\title{
ABSORPTION FILTERS FOR WAVELENGTH TUNING AND FINESSE SWITCHING OF LONG WAVE INFRARED THERMAL DETECTORS
}

\author{
Yuyan Wang, Benjamin J. Potter, Michael Sutton, and Joseph J. Talghader \\ University of Minnesota, Department of Electrical and Computer Engineering \\ Minneapolis, Minnesota 55455
}

\begin{abstract}
A resonant long-wavelength infrared (LWIR) absorption filter for thermal detectors is proposed and demonstrated. The filter operates not by transmitting light like a typical Fabry-Perot, but rather by coupling light in a small wavelength region into one mirror where it dissipates and heats the thermal detector plate. The top plate, or mirror, is movable and can be continuously tuned to have a resonance from 8.7 to $11.1 \mu \mathrm{m}$ using electrostatic actuation with $0-42 \mathrm{~V}$ applied voltage. Since LWIR features are often very course compared to those in the visible, the widths of the tuned resonances are about $1.5 \mu \mathrm{m}$. At an actuation voltage of $45 \mathrm{~V}$, the filter switches into a broadband mode with an absorption width of $2.83 \mu \mathrm{m}$ to enhance sensitivity for scenes with few photons and little spectral information. The switching time between the modes is about $100 \mu \mathrm{sec}$ and the settling time about $400-700 \mu \mathrm{sec}$. Both times are readily compatible with standard microbolometer frame times.
\end{abstract}

\section{INTRODUCTION}

Microfabricated optical cavities are used in a variety of spectrally sensitive devices in the visible, ultraviolet, and near-IR. Currently, telecommunications is probably the biggest application areas, where they are used for resonant cavity-enhanced (RCE) photodetectors $[\mathbf{1}, \mathbf{2}]$, micro machined tunable detectors $[\mathbf{3}, \mathbf{4}]$, and wavelength-division multiplexing systems (WDM) [5, 6], among others. In the middle wavelength infrared (MWIR) and LWIR regions, the fabrication of Fabry-Perot filters are more complex because the optical materials must be infrared-compatible and the layer thicknesses must be larger [7-10].

Despite these successes, it is very difficult to build filters for uncooled LWIR thermal detectors, which has limited previous researchers to demonstrations of discretely tunable 2- or 3-color thermal detectors [11-13], rather than continuously tunable ones. The problem originates with the thermal isolation of the uncooled detector itself. Integrating a tunable Fabry-Perot cavity above a detector will create three air gaps, or 2 air gaps if one places the thermal detector layer inside the cavity. Multiple air gaps mean that there are multiple optical cavities, which may couple together to cause unwanted spectral artifacts and limited tuning.

To negate these problems, we present a single air-gap coupled absorption filter for thermal detectors using a micromachined weakly absorbing top mirror. Instead of filtering in transmission as a standard Fabry-Perot, these devices couple incident radiation into their own top plate, which serves as mirror, absorber, and thermistor all at once. The devices can be continuously tuned, and a tuning range from 8.7 to $11.1 \mu \mathrm{m}$ with $0-42 \mathrm{~V}$ of actuation voltage is demonstrated in this work. Additionally, the devices can also be actuated to a broadband mode at $45 \mathrm{~V}$ where the resonance width is increased to $2.83 \mu \mathrm{m}$. This mode is used to enhance its sensitivity in presence of low-light signals with less spectral information. Experiments have shown that the devices have switching times of about $100 \mu \mathrm{sec}$ and settling times of about $400-700 \mu \mathrm{sec}$, making them compatible with focal plane array frame rates.

\section{DESIGN}

The concepts in this paper apply to all thermal detectors, but in this work, they are demonstrated using a microbolometer. Figure 1 shows a diagram of the microbolometer cavity. The IR absorbing material is deposited on the top of the upper plate of germanium $(\mathrm{Ge})$. A thin layer of chromium $(\mathrm{Cr})$ is chosen as the absorber because it is convenient for deposition and it has the most desirable optical constants of common metals. A key characteristic of $\mathrm{Cr}$ is that it produces a reasonably symmetric resonance with respect to wavelength. The bottom mirror is a modified quarterwave distributed Bragg reflector (DBR), composed of Ge and zinc sulfide $(\mathrm{ZnS})$ on the top of an evaporated gold/chromium $(\mathrm{Au} / \mathrm{Cr})$ reflector with reflectivity centered around $10 \mu \mathrm{m}$.

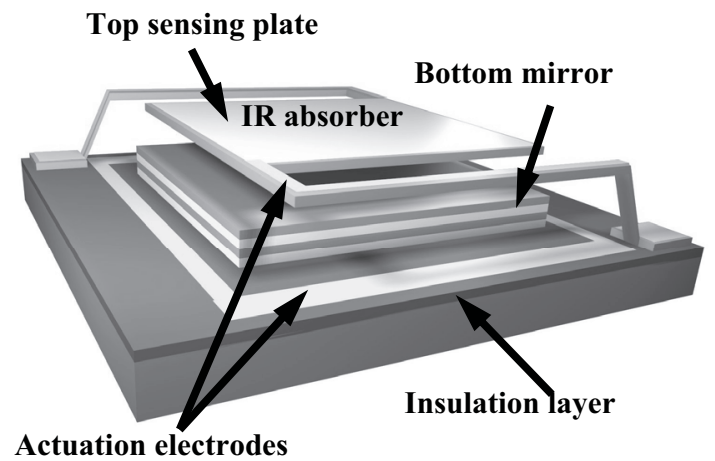

Figure 1. Conceptual diagram of a tunable absorption filter for LWIR microbolometers. The primary actuation electrodes are on the support beams and substrate to delay snap-through and enhance tuning range.

The filter works in two modes. One is broadband IR reflection/absorption detection mode that is designed to maximize the thermal light absorbed. In this mode, a $45 \mathrm{~V}$ actuation voltage pulls the top plate near the bottom mirror, creating a very small gap $(<<\lambda / 10)$. The top mirror itself does not touch the DBR mirror but instead the beam supports make contact so as not to thermally short the device. Although the contact area has not been measured in these devices yet, it is desirable to design the top movable structure such that the contact area is small enough to not affect the thermal performance [14].

The other mode is a reflection/absorption narrowband detection mode, achieved by using $0-42 \mathrm{~V}$ actuation voltage to electronically control the air-gap over a distance of 4.3 to $6.4 \mu \mathrm{m}$. The position of the absorption resonance is continuously tuned, and a sharper resonance is obtained with the non-zero air gap. This mode can be used to recognize objects with subtle differences in 
emissivity spectrum, which are difficult to identify using standard bolometers [15]. Although this paper only describes single devices, the design is compatible for use with a read-out integrated circuit (ROIC) beneath the bottom mirror, which would have the ability to control individual pixels in an array.

The top mirror is designed to have low thermal mass so that the thermal response time is small in the final detector. The resonance width (about $1.5 \mu \mathrm{m}$ in proposed device) matches many coarse spectral features in LWIR. An optimization program based on transmission matrix simulations [16] is used to optimize the layer structure. The optimized results are listed in Table 1.

Table.1 Layer structure of the experimental devices in this study. (The optical constants of these materials have been taken from Palik [17].)

\begin{tabular}{|c|c|c|c|}
\hline \hline Material & Thickness & $\mathbf{n}$ & $\mathbf{k}$ \\
\hline $\mathrm{Cr}$ & $18 \AA$ & 11.8 & 29.8 \\
\hline $\mathrm{Ge}$ & $0.6292 \mu \mathrm{m}$ & 4 & 0 \\
\hline Air gap & $3.7-6.8 \mu \mathrm{m}$ & 1 & 0 \\
\hline $\mathrm{Ge}$ & $0.1480 \mu \mathrm{m}$ & 4 & 0 \\
\hline $\mathrm{ZnS}$ & $0.5557 \mu \mathrm{m}$ & 2.2 & 0 \\
\hline $\mathrm{Ge}$ & $0.2456 \mu \mathrm{m}$ & 4 & 0 \\
\hline $\mathrm{ZnS}$ & $0.5597 \mu \mathrm{m}$ & 2.2 & 0 \\
\hline $\mathrm{Au}$ & $0.50 \mu \mathrm{m}$ & 12.24 & 54.7 \\
\hline $\mathrm{Cr}$ & $300 \AA$ & 11.8 & 29.8 \\
\hline $\mathrm{Si}$ substrate & $\ldots$ & 3.42 & $\ldots$ \\
\hline \hline
\end{tabular}

\section{EXPERIMENTAL DETAILS}

The fabrication of LWIR absorption filters differs from many micromachining processes because of the unusual IR optical materials. The fabrication procedure is illustrated in Figure 2.

First, a silicon nitride layer is deposited with low-pressure chemical vapor (LPCVD) on a four inch n-type (100) silicon wafer. To enhance the bottom mirror reflectivity, a layer of $\mathrm{Au}$ with a seed layer of $\mathrm{Cr}$ is then evaporated on the nitride open areas, followed by two Ge/ZnS quarter-wave pairs (for $\lambda \sim 10 \mu \mathrm{m}$ ) deposited via RF magnetron sputtering. The quarter-wave layers are patterned to form bottom mirrors. The deposition properties of the $\mathrm{Ge}$ and $\mathrm{ZnS}$ are adjusted to get consistently low-stress films and acceptably fast deposition rates. $\mathrm{CF}_{4}$ and $\mathrm{H}_{2}$ plasma are used to dry etch the Ge and $\mathrm{ZnS}$ layers respectively. After completing the substrate reflector, $\mathrm{Au}$ and $\mathrm{Cr}$ layers are deposited to form the electrode lines that surround the bottom mirrors. A sacrificial layer of $\mathrm{SiO}_{2}$ with nominal thickness of $6.8 \mu \mathrm{m}$ is then deposited, which will later form the air gap. A polyimide sacrificial etch was originally attempted and abandoned later because it required a pure-oxygen etch-release that caused too many defects in the optical films. After patterning the anchor holes for the supports, the top-plate structural Ge layer is sputtered, followed by a thin evaporated Cr metal (patterned using lift-off) for absorption. Next, $\mathrm{Au}$ and $\mathrm{Cr}$ films are evaporated along the supporting beams and bonding pads to provide electrical contacts to the top plate for actuation. Finally, the devices are released in a diluted buffered oxide etchant and dried with a critical point dryer to prevent stiction.

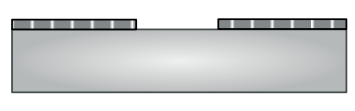

Grow and pattern LPCVD

$\mathrm{Si}_{3} \mathrm{~N}_{4}$ insulation layer

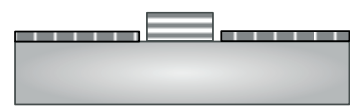

Deposit and pattern bottom mirror

Evaporate bottom actuation electrodes $\mathrm{Au} / \mathrm{Cr}$

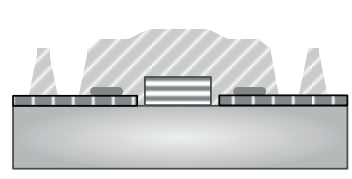

Grow and pattern PECVD $\mathrm{SiO}_{2}$ sacrificial layer

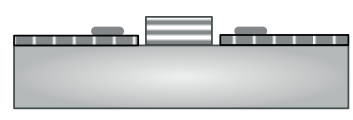

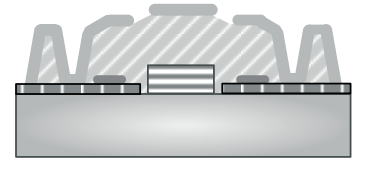

Sputter Ge top plate

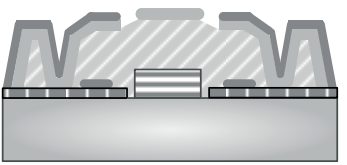

Evaporate top actuation electrodes

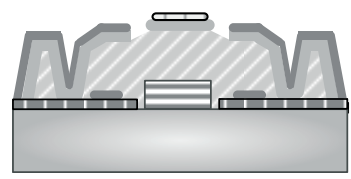

Evaporate Cr for IR absorption

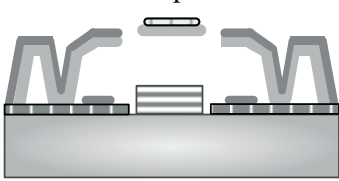

Release device

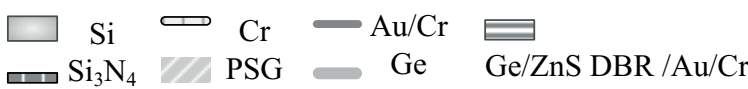

Figure2. Process flow for the LWIR wavelength tunable microbolometer filter.

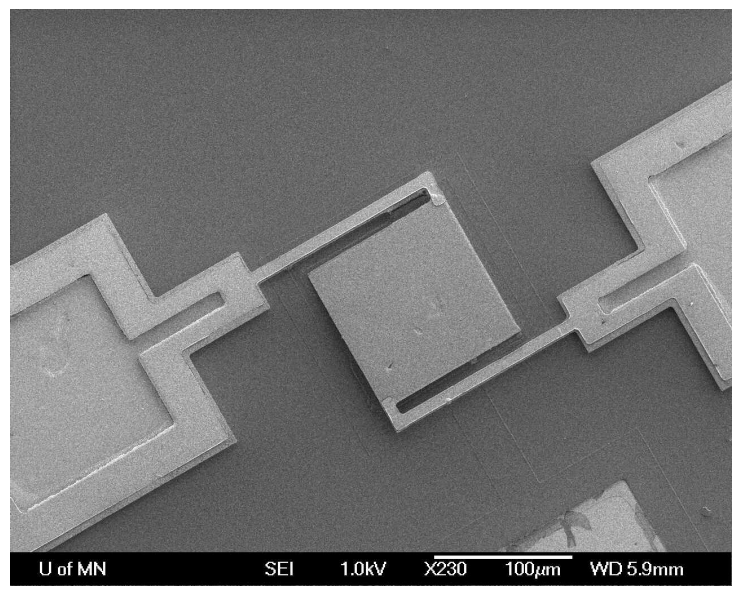

Figure 3. SEM image of a device after etch release. The top plate is 100 by $100 \mu \mathrm{m}$ and is supported by two 150 by $5 \mu \mathrm{m}$ beams. $\mathrm{Au} / \mathrm{Cr}$ has been evaporated on the beams and anchors for actuation. The two structures to the left and right are bonding pads for later testing.

Figure 3 shows a SEM micrograph of a typical finished filter pixel. The top plate flatness deviation is less than $\lambda / 16$ of the design wavelength $(\lambda \sim 10 \mu \mathrm{m})$. The temperature during fabrication (after finishing the initial silicon nitride insulation layer) does not exceed $200^{\circ} \mathrm{C}$, making the process compatible with read-out integrated circuits (ROIC) beneath the bottom mirror. 


\section{MEASUREMENTS AND SIMULATION}

The areas of the filters varied from $25 \times 25 \mu \mathrm{m}^{2}$ to $120 \times 120 \mu \mathrm{m}^{2}$. The measured resistance of the top plates is about $35 \mathrm{~K} \Omega$ at room temperature. The $\mathrm{Cr} / \mathrm{Ge}$ plate has a temperaturesensitive resistance with a coefficient of approximately $0.07 \% / \mathrm{K}$.

A spectrum simulation, which incorporates the fabricated structure thickness and the mirror profile parameters measured by a Zygo interferometric microscope, has been carried out in to determine the air gap spacings that correspond to each wavelength. Figure 4 shows the evolution of the reflected peaks at different air gaps. Note that since there is no transmission, the absorption spectrum is $1-R$. Also, the simulation indicates that nearly all light absorption happens within the top Cr layer on the device, which is necessary for efficient thermal detection.

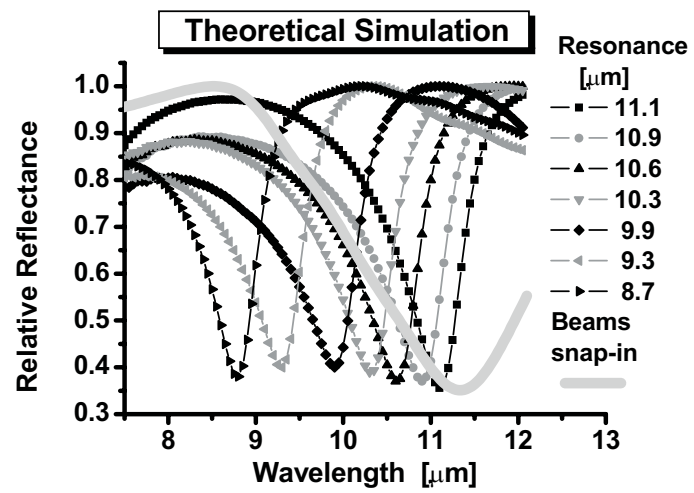

Figure 4. Spectral simulation of the experimental devices. This simulation includes the layer structure of Table 1 and a small curvature present in the top plate that broadens the resonance

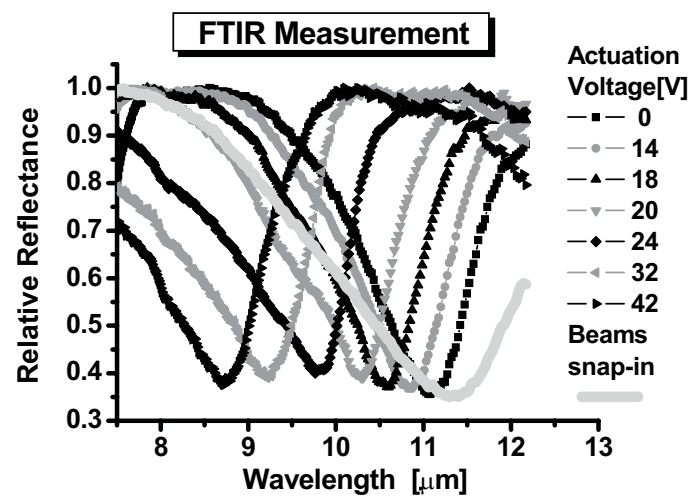

Figure 5. FTIR measurement of the relative reflectance spectra of a 120 by $120 \mu m$ square filter. As the applied voltage is varied from $0-42 \mathrm{~V}$, the narrowband resonance shifts from $11.1 \mu \mathrm{m}$ to $8.7 \mu \mathrm{m}$. A broadband resonance centered at $11.3 \mu \mathrm{m}$ is created when the voltage is increased to $45 \mathrm{~V}$.

The relative reflectance of the $120 \times 120 \mu \mathrm{m}^{2}$ prototype is measured using a Nicolet Magna 750 Fourier Transform Infrared Spectroscopy (FTIR) system with microscope attachment. The filter does not transmit measurable light through the substrate. During the FTIR measurement, a voltage is applied on the supporting arms and the surrounding electrodes beneath the arms using a Keithley 230 Programmable Voltage Source. Changing the voltage level shifts the interference peak, enabling continuous tuning. The voltage-wavelength relationship is stable and shows no hysteresis.

The measured spectrum matches well with simulation and clearly illustrates that the filter has wide continuous tunability. Specifically, the devices are tunable from 8.7 to $11.1 \mu \mathrm{m}$ using 0 $42 \mathrm{~V}$ of actuation voltage. A broadband absorption mode is reached at $45 \mathrm{~V}$, where the resonance full-width-at-half-maximum (FWHM) increases to $2.83 \mu \mathrm{m}$, compared with approximately $1.5 \mu \mathrm{m}$ in the narrowband operation range. The switching between these two modes is emphasized in Figure 6.

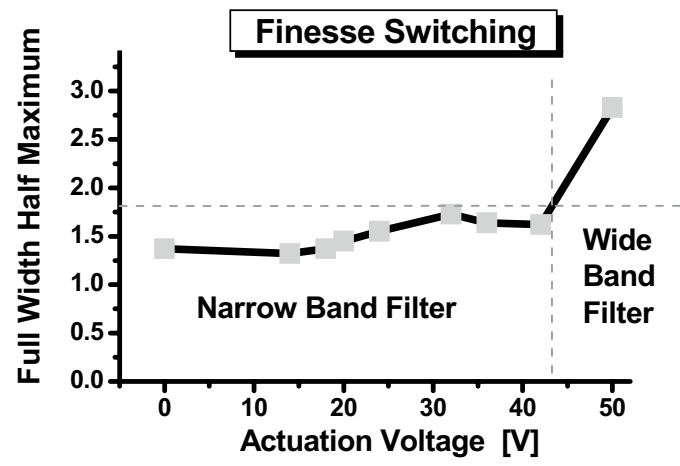

Figure 6. The resonance FWHM versus actuation voltage for the same device tested in Figure 5. The FWHM changes by approximately $87 \%$ between the tuning and broadband modes. The switch is reversible and occurs at about $45 \mathrm{~V}$.

To study the transient response of the filter at the onset of actuation, a position sensitive detector (PSD) is used to capture the movement of the top filter plate under a square-wave actuation voltage. The voltage pulse and the output of PSD are measured simultaneously using a digital oscilloscope. The slowest devices are those with the biggest plates, having dimensions of 120 by $120 \mu \mathrm{m}$. Figure 7 shows a rise time of $110 \mu \mathrm{sec}$ with a settling time about $466 \mu \mathrm{sec}$, and a fall time of $96 \mu \mathrm{sec}$ with a settling time of $672 \mu \mathrm{sec}$ respectively. These transients are easily compatible with microbolometer thermal time constants, which are usually in the range of $5-20 \mathrm{~ms}$.

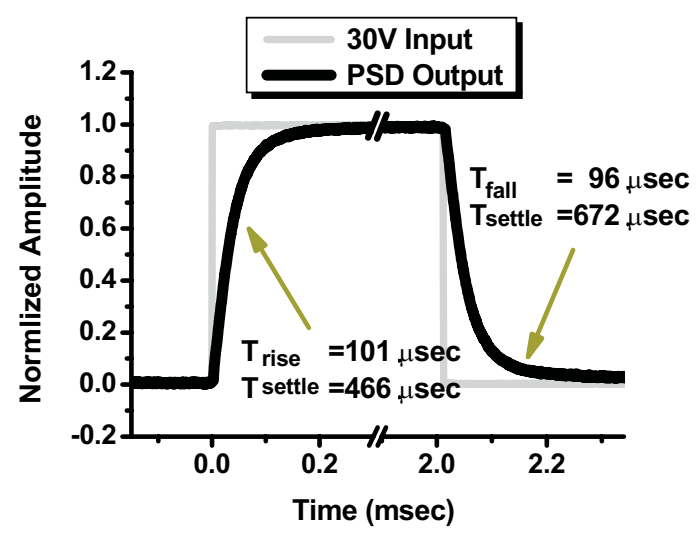

Figure 7. A typical transient response of the devices with plates of size $120 \times 120 \mu \mathrm{m}$. The plates are supported by two beams with lateral size of 100 by $5 \mu \mathrm{m}$ and actuated by a $30 \mathrm{~V}$ square wave voltage pulse. 


\section{CONCLUSIONS}

The electrostatic actuation and optical response of a wavelength tunable absorption filter for thermal detectors has been presented. The filter operates by coupling light over small band of wavelengths into its top mirror, which doubles as the detector plate of a microbolometer. Narrowband tuning from 8.7 to $11.1 \mu \mathrm{m}$ is obtained by varying the actuation voltage from 0 to $42 \mathrm{~V}$, and a broadband mode is obtained at $45 \mathrm{~V}$. Optical characterization has confirmed a good agreement between numerical simulations and experimental results in terms of resonances position and displacement. However, non-ideal features of the fabricated filter can be observed in the broadening and the decreasing amplitude of the reflected peaks.

\section{ACKNOWLEDGEMENT}

This project is supported by Microsystems Technology Office of DARPA and the Army Research Office under contract DAAD19-03-1-0343. The processing was performed at the Nanofabrication Center at the University of Minnesota, which is part of the NSF-sponsored NNIN.

\section{REFERENCES}

[1] R. Kuchibhotla, A Srinivasan, J. C. Campbell, C. Lei, D. G. Deppe, Y. S. He, and B. Streetman, "Low-voltage high-gain resonant-cavity avalanche photodiode," IEEE Photon. Technol. Lett., 3, 354 (1991).

[2] K. Kishino, S. Unlu, J.-I. Chyi, J. Reed, L. Arsenault, and H. Mo, "Resonant cavity-enhanced (RCE) photodetectors," IEEE J. Quantum Electron., 27, 2025 (1991).

[3] E. C. Vail, M. S. Wu, G. S. Li, W. Yuen, and C. J. ChangHasnain, "A Novel Widely Tunable Detector with Wavelength Tracking," Postdeadline Paper, Proceedings of Optical Fiber Communications Conference (OFC), Feb./26-Mar./3, San Diego, California (1995), pp18-2

[4] M. C. Larson, B. Pezeshki, and J. S. Harris, "Vertical coupled-cavity microinterferometer on GaAs with deformablemembrane top mirror", IEEE Photon. Technol. Lett., 7, 382 (1995).

[5] J. Peerlings, A. Dehe, A. Vogt, M. Tilsch, C. Hebeler, Langenham F, P. Meissner, H. L. Hartnagel, "Long resonator micromachined tunable GaAs-AlAs Fabry-Perot filter," IEEE Photon. Technol. Lett., 9, 1235 (1997).

[6] S. Irmer, J. Daleiden, V. Rangelov, C. Prott, F. Romer, M. Strassner, A. Tarraf, H. Hillmer, "Ultralow biased widely continuously tunable Fabry-Perot filter," IEEE Photon. Technol. Lett., 15, 434 (2003).

[7] D. Rossberg, "Silicon micromachined infrared sensor with tunable wavelength selectivity for application in infrared spectroscopy", Sensors and Actuators A: Physical, 47, 413 (1995).

[8] H. Alause, F. Grasdepot, J. P. Malzac, W. Knap and J. Hermann, "Micromachined optical tunable filter for domestic gas sensors", Sensors and Actuators B: Chemical, 43, 18 (1997).

[9] Mitsunori Saito and Hideki Furukawa, "Infrared tunable filter by the use of electrostatic force", Appl. Phys. Lett. 79, 4283 (2001).

[10] JT., Daly, W. A. Bodkin, W. J. Schneller, R. B. Kerr, J. Noto,
R. Haren, M. T. Eismann, B. K. Karch, "Tunable narrow-band filter for LWIR hyperspectral imaging", Proceedings of SPIE Vol 3948 Photodetectors: Materials and Devices V, 1/26-28/00, San Jose, California (2000), pp. 104-115.

[11] S.-W. Han and D. P. Neikirk, "Design of infrared wavelength-selective microbolometers using planar multimode detectors," Proceedings of the SPIE Vol. 5836 Smart Sensors, Actuators, and MEMS II, Microtechnologies for the New Millennium 2005, 05/9-11/05, Seville, Spain (2005), pp. 549-557.

[12] Vladimir N. Leonov and Donald P. Butler, "Two-color thermal detector with thermal chopping for infrared focal-plane arrays", Applied Optics, 40, 2601, (2001).

[13] Y. Wang, B. Potter, M. Sutton, R. Supino and J. Talghader, "Step-wise Tunable Microbolometer Long-wavelength Infrared Filter", Technical digest of the 13th International Conference on Solid-State Sensors, Actuators and Microsystems, 6/5-9/05, Seoul, Korea (2005), pp.1006-1009.

[14] W. B. Song, M. Sutton, and J. J. Talghader, "Thermal contact conductance of actuated interfaces”, Appl. Phys. Lett. 81, 1216 (2002).

[15] R. A. Wood, "Monolithic silicon microbolometer arrays," in Uncooled Infrared Imaging Systems and Arrays, New York Academic, (1997), Semiconductors and Semimetals, 47, ch. 3, pp. 43-121.

[16] H. A. Macleod, Thin Film Optical filters. $3^{\text {rd }}$ ed. Inst of Physics Pub Inc, (2001).

[17] E. Palik, Handbook of optical constants. Academic Press, (1998). 